Check for updates

Cite this: Phys. Chem. Chem. Phys., 2021, 23, 27159

Received 27th February 2021, Accepted 15th November 2021

DOI: 10.1039/d1cp00909e

rsc.li/pccp

\title{
Mechanical behaviour of inorganic solid-state batteries: can we model the ionic mobility in the electrolyte with Nernst-Einstein's relation?
}

\author{
Mei-Chin Pang, (D) Monica Marinescu, Huizhi Wang and Gregory Offer (D)*
}

\begin{abstract}
Inorganic solid-state lithium-metal batteries could be the next-generation batteries owing to their nonflammability and higher specific energy density. Many research efforts have been devoted to improving the ionic conductivity of inorganic solid electrolytes. For a wide range of electrolytes including liquid and solid polymer electrolytes, an independent measurement or calculation of both electrolyte conductivity and diffusion coefficient is often time-consuming and challenging. As a result, NernstEinstein's relation has been used to relate the ionic conductivity to ionic diffusivity after the determination of either parameter. Although Nernst-Einstein's relation has been used for different electrolytes, we demonstrate in this perspective that this relation is not directly transferable to describe the ionic mobility for many inorganic solid electrolytes. The fundamental physics of Nernst-Einstein's relation shows that the relationship between the diffusion coefficient and electrolyte conductivity is derived for ionic mobility in a viscous or a gaseous medium. This postulation contradicts state-of-the-art experimental studies measuring the mechanical behaviour of inorganic solid electrolytes, which show that inorganic solid electrolytes are usually brittle rather than viscoelastic at ambient room temperature. The measurement of loss tangent is required to justify the use of Nernst-Einstein's relation. The outcome of such measurement has two implications. First, if the loss tangent of inorganic solid electrolytes is less than unity in the range of batteries operating temperatures, the impacts of using Nernst-Einstein's relation in modelling the ionic mobility should be quantified. Secondly, if the measured loss tangent is comparable to that of solid polymers and lithium metal, inorganic solid electrolytes may behave in a viscoelastic manner as opposed to the brittle behaviour usually suggested.
\end{abstract}

\section{Introduction}

The inorganic solid electrolyte is a unique class of electrolyte that promises a lower risk of flammability and higher specific energy density when combined with a Li negative electrode. ${ }^{1,2}$ Examples of inorganic solid electrolytes include Lithium Phosphorus OxyNitride (LiPON), cubic-phase $\mathrm{Li}_{7} \mathrm{La}_{3} \mathrm{Zr}_{2} \mathrm{O}_{12}$ (c-LLZO), $\mathrm{Li}_{1+x} \mathrm{Al}_{x} \mathrm{Ge}_{2-x}\left(\mathrm{PO}_{4}\right)_{3}$ (LAGP), $\mathrm{Li}_{2} \mathrm{~S}-\mathrm{P}_{2} \mathrm{~S}_{5}$ (LPS) and $\mathrm{Li}_{10} \mathrm{GeP}_{2} \mathrm{~S}_{12}$ (LGPS). Unlike liquid electrolytes, which usually have an area specific impedance in the range of $\mathrm{m} \Omega \mathrm{cm}^{2}$ and $\Omega \mathrm{cm}^{2}$, the impedance of current inorganic solid electrolytes can be several orders of magnitude higher. ${ }^{3-8}$ Therefore, increasing the conductivity of inorganic solid electrolytes to achieve an ionic conductivity comparable to that of liquid electrolytes $\left(10^{-2} \mathrm{~S} \mathrm{~cm}^{-1}\right)$ has become an important research target. ${ }^{7-13}$ For a wide range of electrolytes, the ionic conduction often

Electrochemical Science \& Engineering, Department of Mechanical Engineering, Imperial College London, SW7 2BP London, UK.

E-mail: gregory.offer@imperial.ac.uk depends on the ionic diffusion coefficient. A higher diffusion coefficient increases the electrolyte conductivity. However, an independent measurement of both electrolyte conductivity and diffusion coefficient is challenging. Therefore, many existing studies use Nernst-Einstein's relation to estimate the ionic conductivity after the determination of the diffusion coefficient or vice versa.$^{13-23}$ Nevertheless, the validity of this relation is questionable in describing the ionic mobility for many inorganic solid electrolytes.

In general, Nernst-Einstein's model relates the diffusion coefficient of an uncorrelated ion to the electrolyte's ionic conductivity. The failure of Nernst-Einstein's relation in modelling the ionic mobility was demonstrated by Marcolongo and Marzari. ${ }^{25}$ Due to the high concentration of mobile ionic species in solid electrolytes, they showed that ionic correlations could act to increase the cooperative ionic motion and subsequently the ionic conductivity. ${ }^{25,26}$ Therefore, the application of Nernst-Einstein's model without considering the ionic interactions leads to an underestimation of the ionic conductivity. ${ }^{25} \mathrm{As}$ a result, the Haven's ratio or the collective correlation factor 
were proposed to account for the ion-ion correlations. ${ }^{26}$ However, these studies have not considered the physical meaning of the forces governing ionic mobility. How would the correlation factor change if the fundamental forces determining the ionic mobility for many inorganic solid electrolytes deviate from those assumed in the derivation of Nernst-Einstein's relation?

By revisiting the original derivations proposed by Einstein ${ }^{27}$ and later expounded by Brett et al., ${ }^{28}$ Feynman et $a .^{29}$ and Peskir, ${ }^{30}$ we study the fundamental assumptions of the Einstein's and Nernst-Einstein's relations as well as their validity in describing the ionic mobility for inorganic solid electrolytes. Despite the popular applications of these two relations in a wide range of electrolytes, in the absence of viscoelastic measurements as a function of temperatures, Nernst-Einstein's relation may not be directly transferable to model the ionic mobility for many inorganic solid electrolytes.

\section{Fundamental physics of Nernst- Einstein's relation}

The Einstein's relation shows that the diffusion coefficient, $D$, is directly proportional to the mobility, $\mu\left[\left(\mathrm{m}^{2} \mathrm{~mol}\right)(\mathrm{J} \mathrm{s})^{-1}\right]:{ }^{31}$

$$
D=\mu R_{\text {gas }} T,
$$

where $R_{\text {gas }}$ is the universal gas constant and $T$ is the absolute temperature. By considering a displacement distribution function, the mean displacement of a suspended particle can be expressed as a function of the diffusion coefficient $\mathrm{via}^{32}$

$$
\overline{x^{2}}=2 D t
$$

where $x$ represents the vector displacement of an atom, and $\overline{x^{2}}$ denotes the mean displacement over all possible routes in time $t$. Eqn (2) was also derived by Smoluchowski and therefore, this equation is known as Einstein-Smoluchowski relation. ${ }^{32}$ The relationship between $\overline{x^{2}}$ and $t$ was later verified experimentally by Perrin, who measured the sedimentation equilibrium in a colloidal suspension. ${ }^{32,33}$ Experiments were performed with different colloidal materials and solvent viscosities under various external conditions to demonstrate the kinetic theory of fluids. ${ }^{33}$

The diffusion coefficient, $D$, can be further related to the equivalent electrolyte conductivity, $\lambda\left[\left(\mathrm{S} \mathrm{cm}^{2}\right)\right.$ equiv. $\left.{ }^{-1}\right]$, through Nernst-Einstein's relationship given by ${ }^{31}$

$$
\lambda=\frac{|z| F^{2} D}{R_{\text {gas }} T} .
$$

It should be noted that eqn (3) is only valid for non-interacting particles, i.e. when the ionic hopping are completely random. ${ }^{34}$ If the hopping probability depends on the direction of the previous hops, the diffusion coefficient becomes correlated. ${ }^{26,34}$ In this case, eqn (3) can be expressed as ${ }^{34}$

$$
\lambda=\frac{|z| F^{2} D}{R_{\mathrm{gas}} T}\left(\frac{\partial \ln c}{\partial \mu}\right),
$$

where $c$ is the site fraction and $\mu$ is the chemical potentials of the particle.

The detailed derivations of the Einstein's, Einstein-Schmoluchowski and Nernst-Einstein's relation can be found in the works of Brett and co-worker, ${ }^{28}$ Feynman et al., ${ }^{29}$ Islam $^{32}$ and Peskir. $^{30}$

\subsection{Governing forces in Nernst-Einstein's relation}

Einstein $^{27}$ developed the theory of diffusion to describe the mobility of small particles in a fluid, where the fluid indicates either a liquid or a gas. The term diffusion denotes the collective motion of all Brownian particles in the fluid. ${ }^{27}$ Einstein determined the diffusion coefficient of a suspended single particle from the dynamic equilibrium condition, which considers the superposition of two processes in opposite directions. In the absence of an external electric field, the first process describes the movement of a suspended single particle under the influence of a force, whereas the second process describes the diffusion due to the thermal molecular movement. ${ }^{27}$ The force considered in Einstein's model is the viscous force, $\vec{F}^{27}$

$$
\vec{F}=6 \pi \eta_{\mathrm{v}} r v,
$$

where $r[\mathrm{~m}]$ is the radius of the particle, $\eta_{\mathrm{v}}[\mathrm{Pa} \mathrm{s}]$ is the dynamic viscosity of the fluid and $v\left[\mathrm{~m} \mathrm{~s}^{-1}\right]$ is the velocity vector of the single particle. The dynamic viscosity is a measure of the fluid resistance to flow (see the bottom left panel of Fig. 1). By considering the dynamic equilibrium of the thermal molecular movement and molecular drift due to viscous force, Einstein demonstrated that the diffusion coefficient depends on the fluid viscosity and the size of suspended particle. ${ }^{27}$

In the presence of an external electric field, the backward viscous force is counterbalanced by the forward electric force: ${ }^{28}$

$$
\underbrace{6 \pi \eta_{\mathrm{v}} r v}_{\text {Viscous force }}=\underbrace{z e E_{\mathrm{F}}}_{\text {Electric force }},
$$

in which $z e$ is the charge of each ion and $E_{\mathrm{F}}$ is the applied electric field between two metallic plates. The top left panel of Fig. 1 illustrates the mobility of an isolated ion in an electric field between two parallel electrodes, where the movement of the ion is governed by a forward electric force and a backward viscous force. Therefore, the drift velocity of the single-particle can be described by ${ }^{28}$

$$
v=\underbrace{\frac{z e}{6 \pi \eta_{\mathrm{v}} r}}_{u} E_{\mathrm{F}},
$$

where $u\left[\left(\begin{array}{ll}\mathrm{C} & \mathrm{m}\end{array}\right)(\mathrm{N} \quad \mathrm{s})^{-1}\right]$ is the proportionality coefficient between the velocity and electric field strength. $u$ can be related to the ionic mobility, $\mu$, from eqn (1) via $\mu=u /(z F)$, where $F$ is the Faraday's constant.

\subsection{Diffusion coefficients in gaseous media}

For gaseous media involving the acceleration and collisions of gaseous molecules, Feynman et $a .^{29}$ demonstrated that the drift velocity of the gas particles could be derived by 


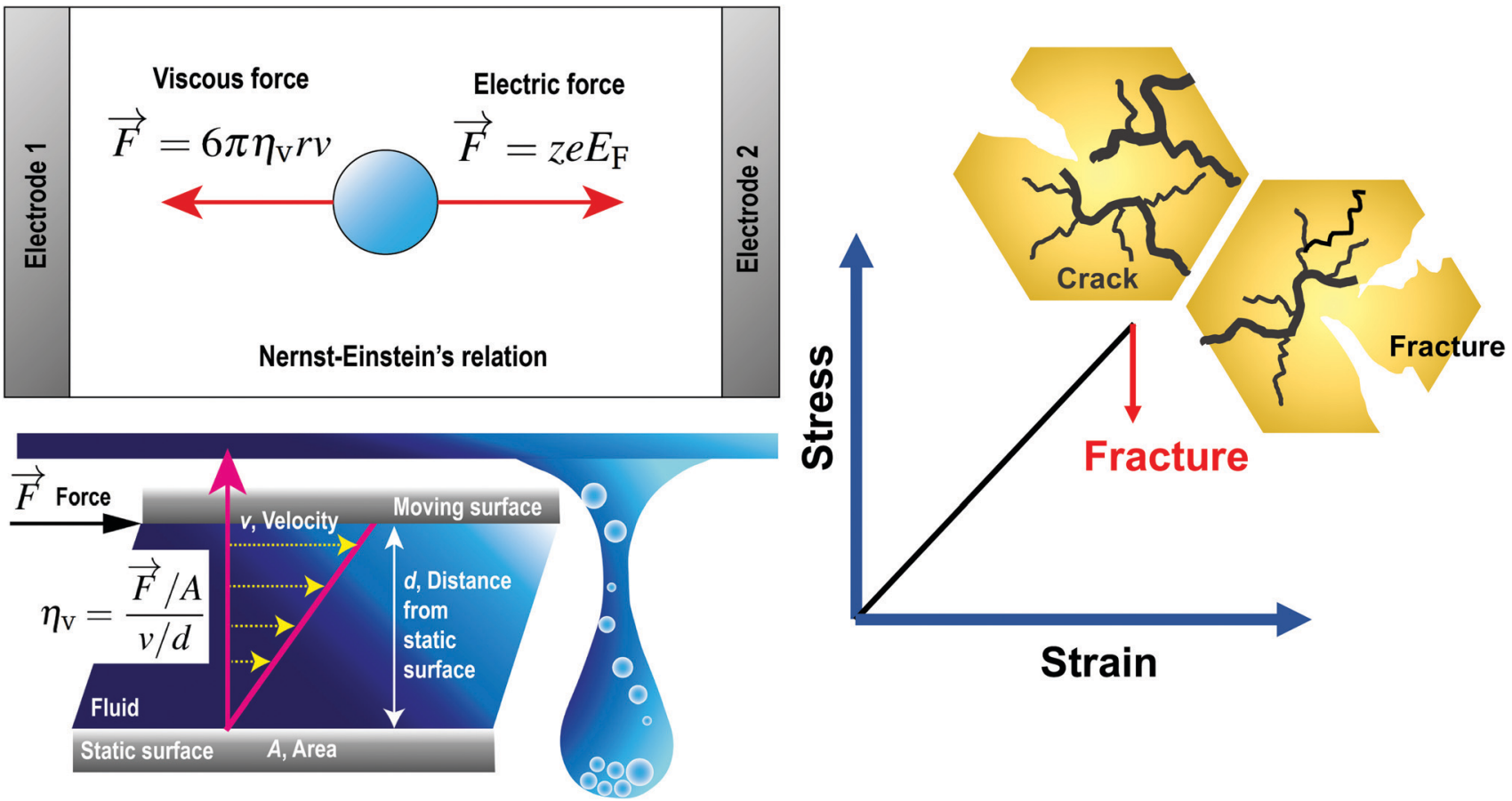

Fig. 1 (Top left) Governing forces given by Nernst-Einstein's relationship, where the forward electric force is in a dynamic equilibrium with the backward viscous force. (bottom left) The dynamic viscosity is a measure of the fluid resistance to flow. (right) For brittle inorganic solid electrolytes characterised by cracks formation and electrolyte fracture, the stress-strain relationship before the electrolyte fracture can be approximated as linear. Reproduced with permission from ref. 24, Copyright (2021), CC-BY.

considering the drift due to an externally applied force, $\vec{F}\left[(\mathrm{~kg} \mathrm{~m}) \mathrm{s}^{-2}\right]$. In this case, the drift velocity becomes ${ }^{29}$

$$
v=\mu_{\text {gas }} \vec{F}
$$

where $\mu_{\text {gas }}\left[\mathrm{s} \mathrm{kg}^{-1}\right]$ is the constant of proportionality denoted as the gaseous mobility. This equation is derived based on the assumption that gaseous particles accelerate under the applied external force: ${ }^{29}$

$$
\begin{aligned}
v & =a \tau, \\
& =\frac{\vec{F}}{m} \tau,
\end{aligned}
$$

in which $a\left[\mathrm{~m} \mathrm{~s}^{-2}\right]$ is the acceleration of the gaseous particles, $\tau[\mathrm{s}]$ is the mean time between collisions and $m[\mathrm{~kg}]$ is the mass of the gas molecule. By comparing eqn (8) and (9), the gaseous mobility is defined by $\tau / \mathrm{m}$. $\mu_{\text {gas }}$ can be further related to the diffusion coefficient $\left(D=\mu_{\text {gas }} k T\right)$ through the kinetic theory of gases: ${ }^{29}$

$$
\frac{1}{2} m v_{\mathrm{rms}}^{2}=\frac{3}{2} k T,
$$

where $v_{\mathrm{rms}}\left[\mathrm{m} \mathrm{s}^{-1}\right]$ is the root-mean-square velocity of gas molecules and $k\left[\mathrm{~J} \mathrm{~K}^{-1}\right]$ is the Boltzmann's constant $\left(k=R_{\text {gas }} / N_{\mathrm{A}}\right)$. Eqn (10) can be derived from the ideal gas law under the assumption that the volume of gaseous molecules is negligible compared to the total volume, in which the gas is contained.

By examining the fundamental assumptions in these derivations, it is evident that the relationship between the diffusion coefficient and electrolyte conductivity can only be established in a viscous or a gaseous medium.

\subsection{Diffusion measurements in inorganic solid electrolytes}

Hayamizu and Aihara ${ }^{37}$ have measured the diffusion coefficient and ionic conductivity of ${ }^{7} \mathrm{Li}$-ion in sulfide-based solid electrolyte, $\left(\mathrm{Li}_{2} \mathrm{~S}\right)_{7}\left(\mathrm{P}_{2} \mathrm{~S}_{5}\right)_{3}$ using PGSE NMR method and AC impedance spectroscopy independently. By using the PGSE NMR method, the diffusion coefficient can be determined from the Stejskal and Tanner equation, where the echo attenuation depends on the gradient strength, $\mathrm{g}\left[\mathrm{T} \mathrm{m}^{-1}\right]$, interval between the leading edges of the gradient pulse, $\Delta[\mathrm{ms}]$ and pulse duration, $\delta[\mathrm{ms}] .{ }^{37,39,40}$ The ${ }^{7}$ Li-ion diffusion measured by the PGSENMR method is determined by the migration of the ${ }^{7} \mathrm{Li}$-ion during a time interval $\Delta .^{39}$ The first signal encodes the target ${ }^{7} \mathrm{Li}$-ion, and the second signal detects the encoded species after an interval $\Delta \cdot^{39}$ The measured signal attenuations usually have a standard deviation of $2-5 \%$ for ${ }^{7} \mathrm{Li}$-ion. ${ }^{35,36}$

Fig. 2(a) shows the temperature-dependent diffusion coefficient between $303 \mathrm{~K}$ and $353 \mathrm{~K}$. The apparent diffusion coefficient measured by the PGSE NMR method shows a qualitative agreement with the estimated diffusion coefficient from the measured ionic conductivity using Nernst-Einstein's relation (i.e. both measured and estimated diffusion coefficients become larger with increasing temperature). However, the diffusion coefficients measured with the PGSE NMR method deviate significantly from the estimated diffusion coefficient using Nernst-Einstein equation. 


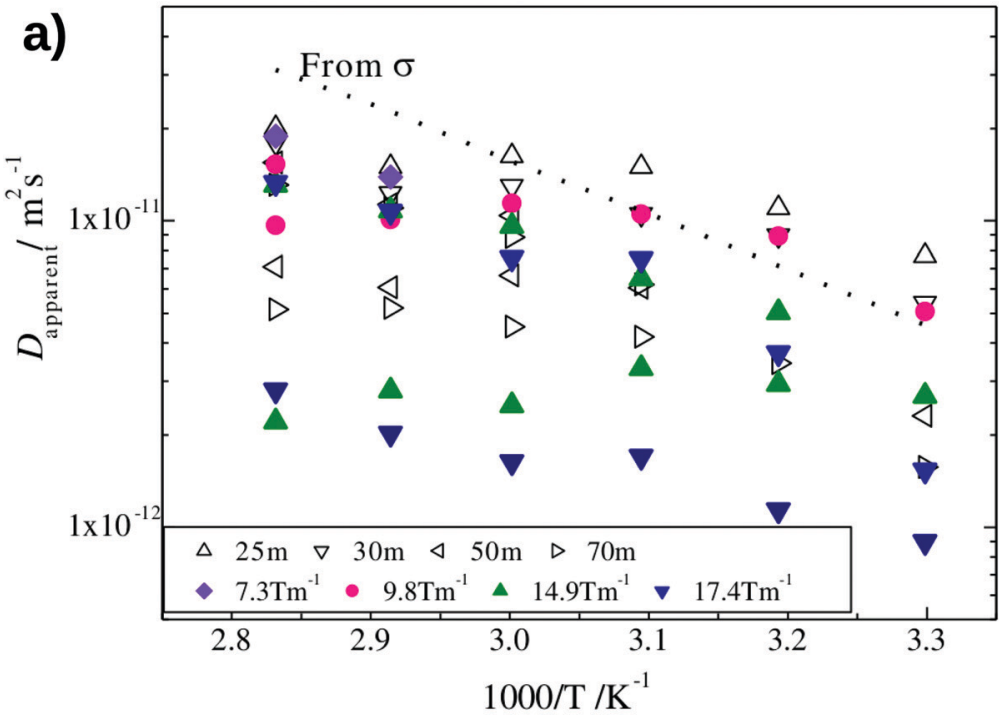

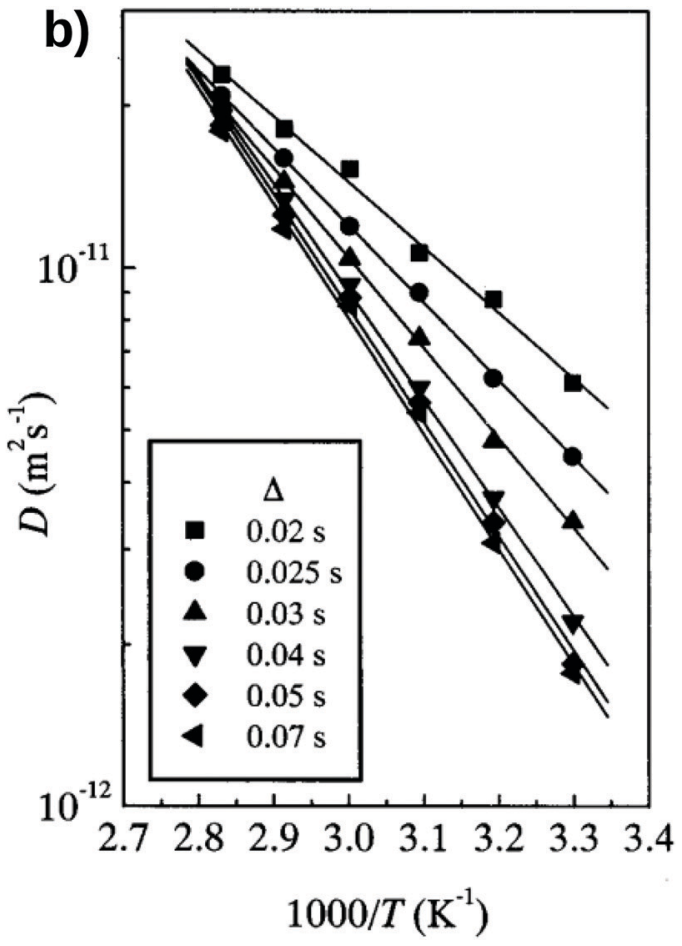

Fig. 2 The degree of scattering observed in the Pulse-Gradient Spin-Echo (PGSE)-NMR diffusion measurements of (a) inorganic solid electrolytes and (b) solid polymer electrolytes. (a) The opened triangles represent the diffusion measurements using different intervals between two gradient pulses, $\Delta$, whereas the filled markers denote the diffusion measurements using different magnitudes of gradient strength, $g$. The standard notation to denote pulse delays of milliseconds $(\Delta)$ is "ms" instead of " $m$ ". The dotted line is the estimated diffusion coefficient from the ionic conductivity measurement using Nernst-Einstein's equation. The standard deviations of a typical ${ }^{7} \mathrm{Li}$-ion PGSE-NMR measurement are between $2-5 \% .{ }^{35,36}$ Reproduced with permission from ref. 37, Copyright (2013), Elsevier. (b) The temperature-dependent diffusion measurement as a function of $\Delta$ in solid polymer electrolytes. Reproduced with permission from ref. 38, Copyright (2000), Elsevier.

Due to the strong dependence on the $g$ - and $\Delta$-values, the PGSE-NMR measurement method shows no unique apparent diffusion coefficient in the sulfide-based solid electrolyte. For instance, Fig. 2(a) shows that at the same gradient strength of $g=14.9 \mathrm{~T} \mathrm{~m}^{-1}$ and the same interval of $\Delta=30 \mathrm{~ms}$, different diffusion coefficients were obtained for all temperatures. ${ }^{37}$ Two modes of a fixed $\delta$ with varying $g$ and a fixed $g$ with varying $\delta$ yield different echo attenuation plots. ${ }^{39}$ Such observations differ from the diffusion measurements taken in polymer electrolytes, organic solvents and ionic liquids, where the measured signal attenuations can be quantified reliably. ${ }^{35,38,41-43}$ Fig. 2(b) shows the diffusion measurement in solid polymer electrolytes. Unlike the strong degree of scattering observed in the diffusion measurement of $\left(\mathrm{Li}_{2} \mathrm{~S}\right)_{7}\left(\mathrm{P}_{2} \mathrm{~S}_{5}\right)_{3}$ inorganic solid electrolytes (Fig. 2a), the temperature-dependent diffusion coefficients do not scatter depending on measuring conditions. ${ }^{38}$ The scattered ionic diffusions in $\left(\mathrm{Li}_{2} \mathrm{~S}\right)_{7}\left(\mathrm{P}_{2} \mathrm{~S}_{5}\right)_{3}$ (for example, the diffusion measurement at the gradient strength $g=9.8 \mathrm{~T} \mathrm{~m}^{-1}$ denoted by the pink circles in Fig. 2a) also reveal that the dependence of diffusion coefficients on ionic conductivity is non-linear. ${ }^{37}$ Such behaviour is different from the estimated diffusion coefficient using Nernst-Einstein's relation (eqn (3)), where the diffusion coefficient is predicted to be linearly proportional to the electrolyte conductivity.
Even with a better-controlled measuring condition, Hayamizu et $a .^{39}$ showed that the simple application of Nernst-Einstein's equation in inorganic solid electrolytes is still questionable. They showed that a PGSE-NMR diffusion measurement with a longer time interval, $\Delta$, could reduce the degree of scattering observed in inorganic solid electrolytes. ${ }^{39}$ However, the diffusion coefficient calculated from NernstEinsteins equation is larger than the equilibrated diffusion coefficient obtained by the PGSE-NMR measurements. ${ }^{39,40}$ As a result, the $N_{\text {carrier }}$ calculated from the experimental ionic conductivity and equilibrated diffusion coefficient through Nernst-Einsteins relation were found to be higher than the values estimated by the molecular formula and experimental density. ${ }^{39,40}$ In addition, an equilibrated measurement of the diffusion coefficient may not be representative of the fast ionic transport across the solid electrolyte.

By comparing the echo-attenuation plots obtained in single crystals and powders, grain morphologies were shown to cause such unusual diffusive behaviour in inorganic solid electrolytes. ${ }^{40}$ Nevertheless, another possible reason could be the lack of viscoelastic behaviour in an inorganic solid electrolyte. In reality, the viscosity, $\eta_{\mathrm{v}}$, is an important parameter determining the ionic diffusion coefficient. ${ }^{35,36,42,43}$ For instance, Hayamizu et al. $^{35}$ showed that the diffusion coefficients of 12 organic solvents are directly correlated to the 


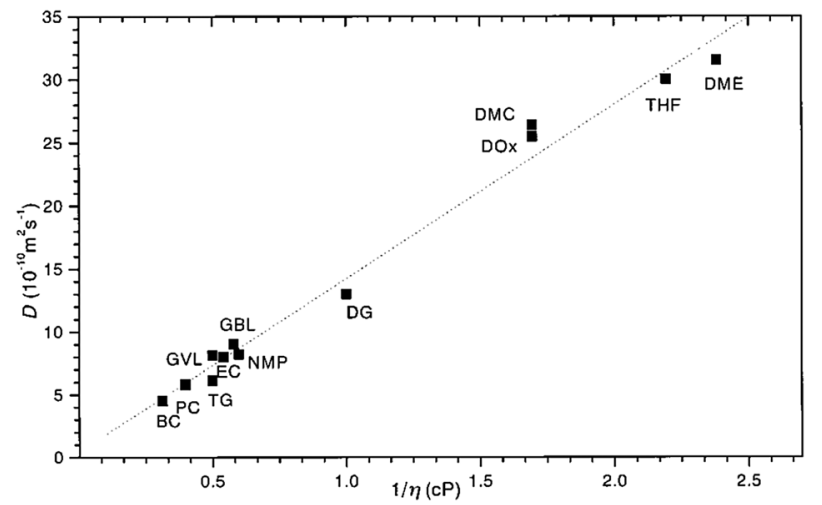

Fig. 3 The relationship between the diffusion coefficient and viscosity of 12 organic solvents. Reproduced with permission from ref. 35, Copyright (1999), American Chemical Society.

inverse of viscosity (Fig. 3). A good correlation can be found between the viscosity diffusion coefficient $\left(D_{\eta}\right)$ and viscosity $\left(\eta_{v}\right)$ according to Stokes-Einstein's equation: ${ }^{35}$

$$
D_{\eta}=\frac{k T}{6 \pi \eta_{\mathrm{v}} r},
$$

where $r$ is the effective hydrodynamic radius.

\section{Mechanical behaviour of inorganic solid electrolytes}

In a broad sense, the family of inorganic solid electrolytes consists of two sub-branches: (i) ceramic-based solid electrolytes and (ii) glassy solid electrolytes. By using NernstEinstein's relation to describe the mobility of $\mathrm{Li}^{+}$ions in inorganic solid electrolytes, one assumes that the transport of $\mathrm{Li}^{+}$ions through inorganic solid electrolytes can be described as viscous flow or viscoelastic behaviour. However, many of the existing experimental results measuring the mechanical behaviour of inorganic solid electrolytes usually indicate that inorganic solid electrolytes are brittle and ceramic-like rather than viscoelastic. $^{44-49}$

\subsection{Characteristics of brittle and viscoelastic solids}

A brittle solid typically exhibits a linear stress-strain relationship with very few plastic deformation signs before a catastrophic crack or fracture. This phenomenon is caused by the low capacity of ceramics in absorbing the applied energy before they fracture.$^{50,51}$ Unlike a viscoelastic solid, stress in a brittle material is independent of the rate of strain. This relationship before failure can be described by Hooke's law as

$$
\sigma_{\mathrm{st}}=E \varepsilon,
$$

in which $\sigma_{\mathrm{st}}[\mathrm{Pa}]$ is the applied stress, $\varepsilon[-]$ is the corresponding strain, and $E[\mathrm{~Pa}]$ is the proportionality constant known as Young's modulus. Fig. 4(a) shows the stress-strain relationship for brittle ceramics such as ZTA, Y-PSZ, Mg-PSZ and Syalon $+\mathrm{BN} .{ }^{50}$ Gogotsi et al. ${ }^{50}$ introduced a brittleness measure, $\chi$, to characterise the elastic behaviour of ceramics. He considered a
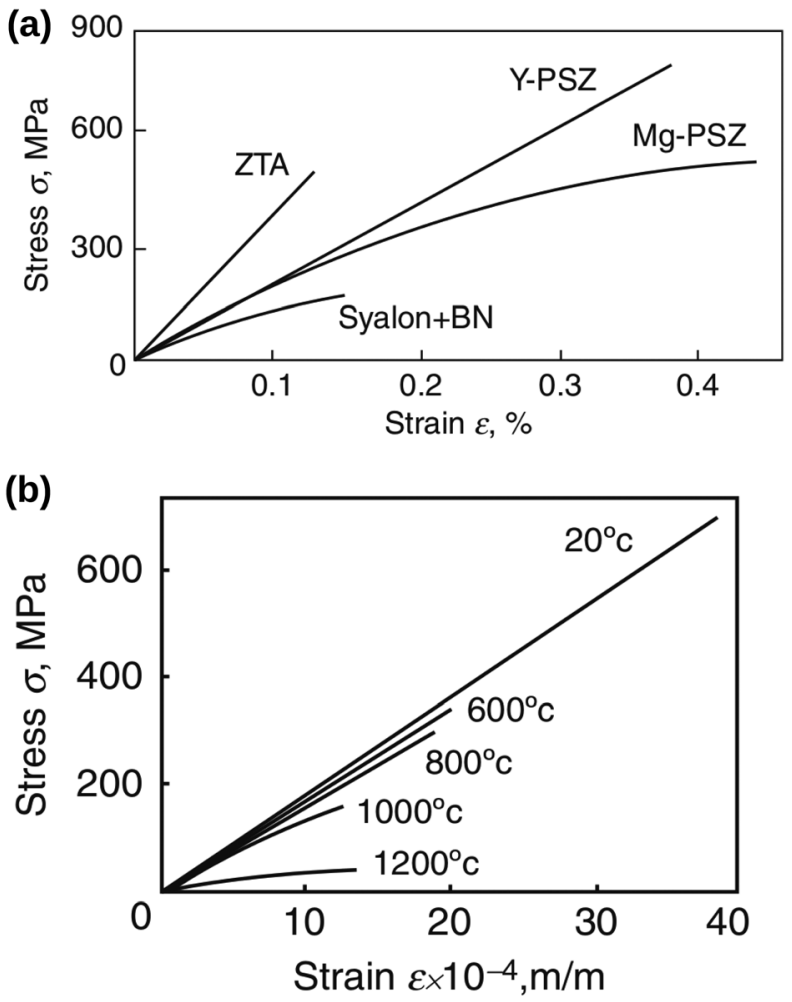

Fig. 4 (a) Illustration of the stress-strain relationship for brittle ceramics: ZTA and Y-PSZ have a brittleness measure of 1, whereas Syalon + BN and Mg-PSZ have a brittleness measure of 0.70 and 0.44 , respectively. (b) The stress-strain relationship for Y-PSZ ceramics at different temperatures. Reproduced from ref. 50, Copyright (2014), with permission from Springer.

ceramic as linearly elastic if the brittleness measure $\chi=1$ or inelastic if $\chi<1$. While materials such as ZTA and Y-PSZ have a $\chi$-value of 1 , he showed that the mechanical behaviour of $\mathrm{Mg}$ PSZ and Syalon + BN can deviate slightly from the linearly elastic regime..$^{50}$ Nevertheless, for a wide range of temperatures, the mechanical behaviour of brittle ceramics can be approximated with Hooke's law, as shown by Fig. 4(b).

In contrast, viscoelastic solids such as polymers and Li metal exhibit mechanical behaviour, in which both solid-like and liquid-like characteristics are prevalent. ${ }^{52-55}$ As a result, these materials can undergo significant deformations before failure (see the inset of Fig. 6d). Stress is always proportional to the rate of strain, indicating a time-dependent deformation or a viscous behaviour. For an ideal viscous material, the stressstrain relationship can be represented by

$$
\sigma_{\mathrm{st}}=\eta_{\mathrm{v}} \frac{\partial \varepsilon}{\partial t}
$$

where $\eta_{\mathrm{v}}$ is the dynamic viscosity - a key parameter influencing the viscous force in eqn (5).

Vincent ${ }^{56}$ illustrated the stress-strain relationships for different mechanical behaviours (Fig. 5). As shown by the top left panel in Fig. 5, an elastic material will return to its original shape on removal of the load. However, due to dissipation of the deformation energy, a plastic material will not return to its original shape after the load is removed (see bottom left panel). 


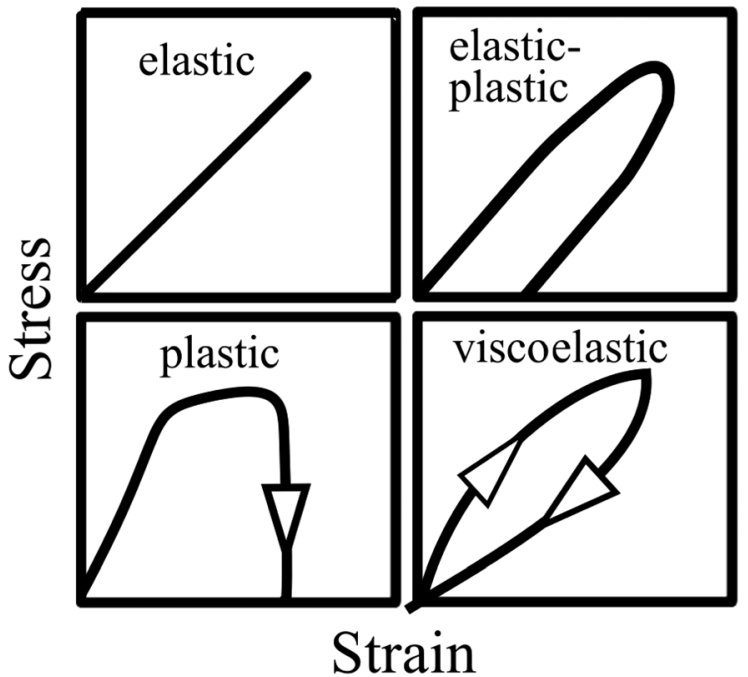

Fig. 5 Illustration of stress-strain relationships for different types of mechanical behaviours: elastic, elastic-plastic, plastic and viscoelastic characteristics. Reproduced with permission from ref. 56, Copyright (2012), Princeton University Press.

While an elastic-plastic mechanical behaviour shows a combination of elasticity and permanent deformation, a viscoelastic material will return to its original shape on removal of the load. Nevertheless, compared to the pure elastic mechanical behaviour, a time-dependent deformation occurs due to the contribution of the viscous component to the viscoelastic response. ${ }^{56}$

\subsection{The effects of operating temperatures}

Temperature plays a significant role in determining the viscoelastic behaviour of a solid. Brittle material such as inorganic solids also have different stress-strain relationships at room temperature compared to at high temperature. While a linear stress-strain relationship is usually observed before an immediate crack or a fracture through the material at ambient room temperature, at high temperatures such as $200-400{ }^{\circ} \mathrm{C}$, the stress-strain relationship has been found to be non-linear even before a crack or fracture. ${ }^{51}$

Lewis et al. $^{49}$ illustrated the crack formation in the LAGP solid electrolyte captured by low-magnification cross-sectional SEM images (see Fig. 6a), when the LAGP cells were cycled until failure at $0.1 \mathrm{~mA} \mathrm{~cm}^{-2}$ and $0.5 \mathrm{~mA} \mathrm{~cm}^{-2}$. As the applied current densities increase, they showed that the horizontal cracks at the interface penetrated deeper into the bulk solid electrolyte. These images portray cracks propagating in the absence of plastic deformation signs, indicating that LAGP is brittle and has a ceramic-like mechanical behaviour. ${ }^{49}$ Typical deformation behaviour is characterised by an irreversible change in volume in response to exerted forces, as portrayed in the inset of Fig. 6(c). In Fig. 6(b), Korte and Clegg ${ }^{51}$ showed the fracture of ceramic micropillars into multiple fragments when compressed with a nano-indenter at room temperature, corroborating the conclusion that ceramic-like electrolyte is brittle. Nevertheless, when the same material was compressed at elevated temperatures such as $200{ }^{\circ} \mathrm{C}$ and $400{ }^{\circ} \mathrm{C}$, considerable deformation rather than a brittle fracture was observed. ${ }^{51}$ In contrast, Fig. 6(c) shows that a viscoelastic solid such as $\mathrm{Li}$ metal deformed significantly even at room temperature when it was compressed. ${ }^{55}$

While high operating temperatures improve the ionic transport across the inorganic solid electrolyte, the temperature increment is capped by the (i) interfacial decomposition at high temperatures and (ii) the low melting point of Li metal. Firstly, due to the low interfacial stability, the rate of decomposition at both interfaces was shown to increase at high temperatures. ${ }^{57-59}$ For instance, Wang et al. ${ }^{57}$ showed that a solid-state cell cycled at $80{ }^{\circ} \mathrm{C}$ lost about $35 \%$ of the initial capacity after 250 cycles while the cell cycled at $25{ }^{\circ} \mathrm{C}$ maintained the same initial capacity. By using TEM and STEM-EELS analysis, they showed that the decrease in the capacity at $80{ }^{\circ} \mathrm{C}$ was caused by the decomposition reaction in the disordered $\mathrm{LiCoO}_{2}$ layer at the interface. ${ }^{57,60}$ Secondly, if pure metallic $\mathrm{Li}$ is used as the negative electrode to boost the gravimetric and volumetric energy density, ${ }^{1}$ one should note that Li melts at $180{ }^{\circ} \mathrm{C}$. This limitation indicates that a solid-state cell with a $\mathrm{Li}$ electrode cannot be operated at temperatures approaching $180{ }^{\circ} \mathrm{C}$. Therefore, within this limited operating temperature range, ceramic-based inorganic solid electrolytes are expected to demonstrate a brittle rather than a viscoelastic mechanical behaviour.

\subsection{Glassy solid electrolytes}

However, due to different chemical compositions, structures and processing conditions, one should also note that the brittleness of inorganic solid electrolytes cannot be generalised for all different solid electrolytes. While solid electrolytes such as c-LLZO, LLTO and LATP have a low fracture toughness comparable to that of brittle inorganic materials $\left(\approx 1 \mathrm{MPa} \mathrm{m}^{-2}\right)$ (see Fig. 7(a)), ${ }^{61}$ Kalnaus et al. ${ }^{61}$ demonstrated recently that glassy solid electrolytes such as LiPON portrayed nanoscale ductility and time-dependent deformation behaviour. By using nanoindentation, they observed accommodation of stress via pile-up rather than by cracking in LiPON, as shown by Fig. 7 b. ${ }^{61}$

Unlike ceramics, glasses consist of network formers such as $\mathrm{SiO}_{2}$ and network modifiers such as alkali oxides. ${ }^{63}$ At high temperatures, a glass-forming melt behaves as a liquid and becomes a glassy solid upon cooling below the glass-transition temperature. ${ }^{64}$ The viscosity of glasses depends strongly on the temperature, which has been usually described by the VogelFulcher-Tamman's relation: ${ }^{65}$

$$
\eta_{\mathrm{v}}(T)=\eta_{0} \exp \left(\frac{D_{\text {frag }} T_{0}}{T-T_{0}}\right)
$$

where $\eta_{0}$ is the constant, $D_{\text {frag }}$ is the fragility index of glasses and $T_{0}$ is the temperature, at which the liquid ceases to flow. Glasses such as soda-lime glasses have very high glasstransition temperature at $840-900 \mathrm{~K}^{66}$ As a result, the viscosity-temperature behaviour was determined for temperatures ranging from $793 \mathrm{~K}$ to $1772 \mathrm{~K}^{66}$

Some glasses like oxide glasses are ionic conductors, which imply that their ionic conductivity can be measured by the impedance spectroscopy. ${ }^{63}$ Mehrer et al. ${ }^{63}$ measured the 

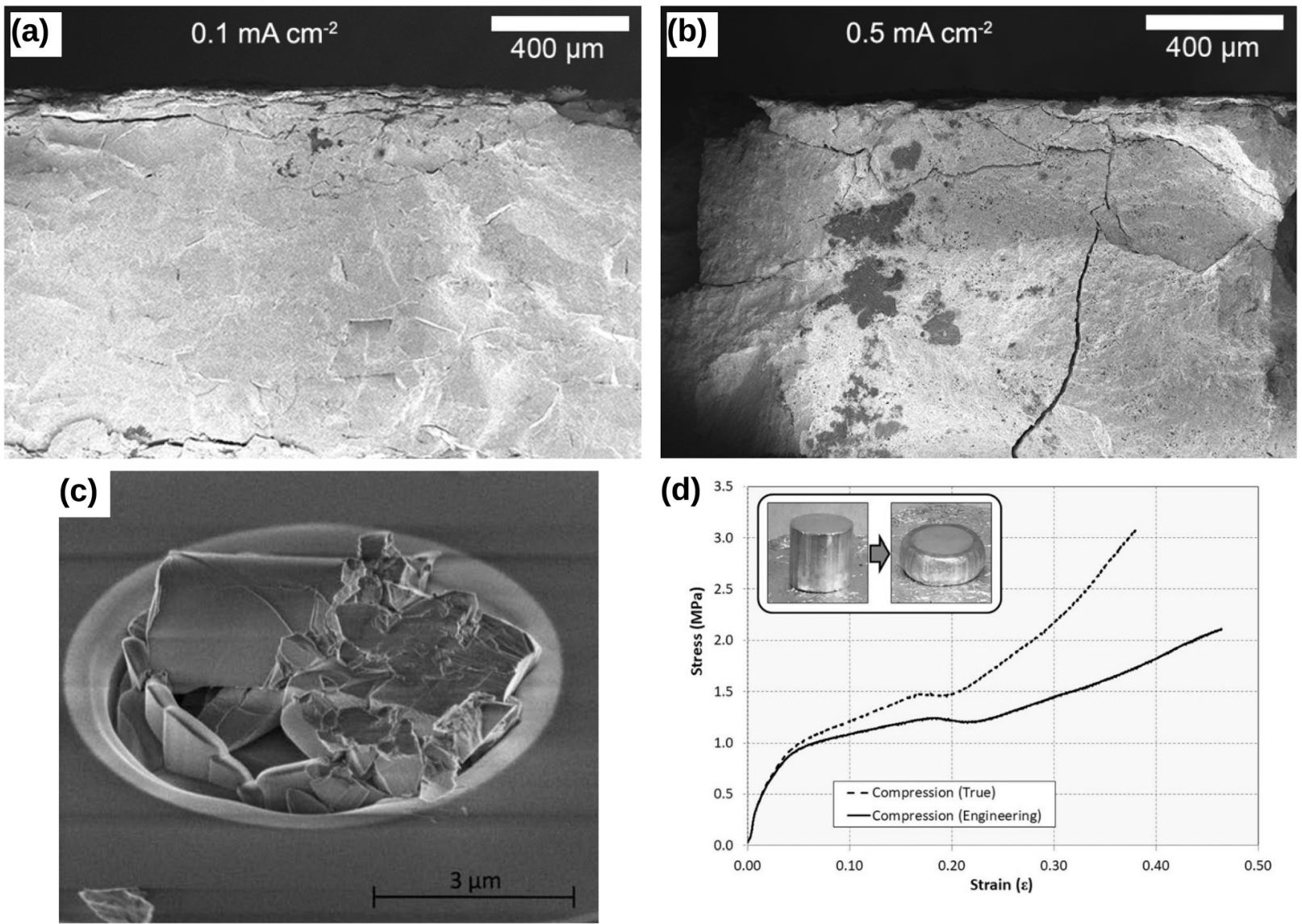

Fig. 6 Comparison of the brittle and viscoelastic material behaviour: cross-sectional SEM images of $\mathrm{Li}_{1+x} \mathrm{Al}_{x} \mathrm{Ge}_{2-x}\left(\mathrm{PO}_{4}\right)_{3}(\mathrm{LAGP}$ solid electrolyte cycled until failure at (a) $0.1 \mathrm{~mA} \mathrm{~cm}^{-2}$ and (b) $0.5 \mathrm{~mA} \mathrm{~cm}^{-2}$ current densities. Reprinted from ref. 49, Copyright (2019), with permission from American Chemical Society. (c) The fracture of ceramic micropillars upon compression with a nano-indenter at room temperature. Reprinted from ref. 51, Copyright (2009), with permission from Elsevier. (d) The compressive stress-strain behaviour of a solid Li at room temperature during a loading cycle. The inset shows that Li metal deforms significantly when subjected to compressive stress, indicating that Li metal could exhibit a viscoelastic mechanical behaviour. $\dagger$ Reprinted from ref. 55, Copyright (2019), with permission from Springer Nature.

temperature-dependent diffusion coefficients of a soda-lime silicate glass for temperatures ranging from approximately $450 \mathrm{~K}$ to $1600 \mathrm{~K}$ (see Fig. 8). In their works, the charge diffusion coefficient or sometimes also denoted as the conductivity diffusion coefficient, $D_{\sigma}$ is the diffusion coefficient determined from Nernst-Einstein's relation, where the particles were assumed to be non-interacting (eqn (3)). They obtained the tracer diffusion coefficient of radioisotopes ${ }^{22} \mathrm{Na}$ and ${ }^{45} \mathrm{Ca}$ with the radiotracer technique. ${ }^{63}$ By comparing the conductivity diffusion coefficient to the tracer diffusion coefficient of ${ }^{22} \mathrm{Na}$ and ${ }^{45} \mathrm{Ca}$ radioisotopes, they concluded that the ionic conductivity of a soda-lime glass is caused predominantly by the mobility of $\mathrm{Na}$ ions. ${ }^{63}$ They also determined the viscosity diffusion coefficient by Stokes-Einstein's relation (eqn (11)), which corresponded to the mobility of the network-forming units. ${ }^{63}$ Nevertheless, as discussed in Section 3.2, operating temperatures ranging from $450 \mathrm{~K}$ to $1600 \mathrm{~K}$ are not feasible for

\footnotetext{
$\dagger$ For a compression test, one should note that the true stress should be smaller than the engineering stress due to a larger instantaneous cross-sectional area during compression.
}

inorganic solid-state Li-metal batteries. Therefore, future works are required to quantify the viscoelastic behaviour of glassy solid electrolytes and the interplay with their ionic conduction mechanisms at ambient temperatures.

\subsection{Loss tangent measurements}

Although the nanoscale micrograph of LiPON reveals the accommodation of stress via pile-up, the value of fracture toughness for LiPON remains unknown. ${ }^{61}$ Moreover, a fracture rather than a deformation was observed in another micrograph of LiPON (Fig. 7c), ${ }^{62}$ thus suggesting that LiPON may have different mechanical behaviour at different length scales. It is also worth noting that not all glassy materials exhibit time-dependent deformations. While metallic glasses are likely to yield plastically under an opening stress, oxide glasses have a very low fracture toughness $\left(<1 \mathrm{MPa} \mathrm{m}^{1 / 2}\right)$ and were shown to exhibit brittle cracks. ${ }^{67,68}$

Since the viscoelastic behaviour is a time- and frequencydependent deformation, the value of the loss tangent can be used as the indication of a viscoelastic characteristic. ${ }^{52,69}$ In a viscoelasticity measurement, the material is perturbated with a small oscillating strain and the resulting stress is measured. ${ }^{56}$ 
(a)

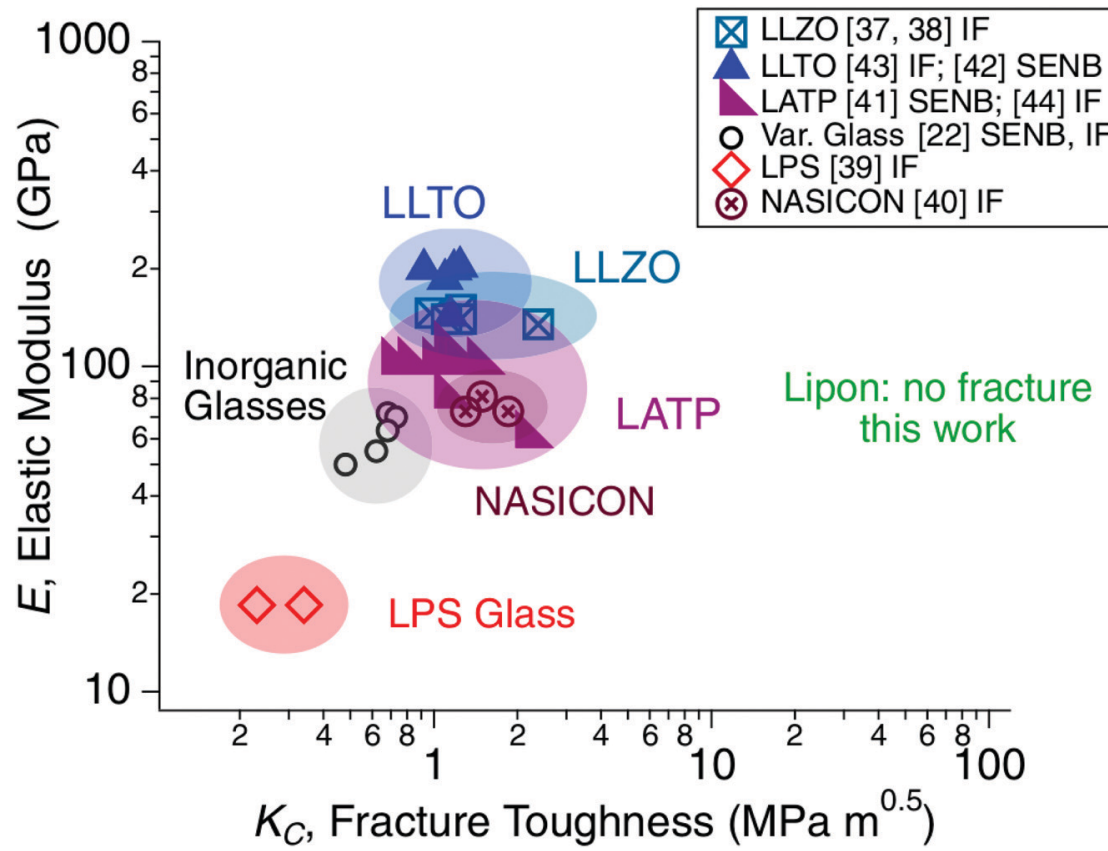

(b)

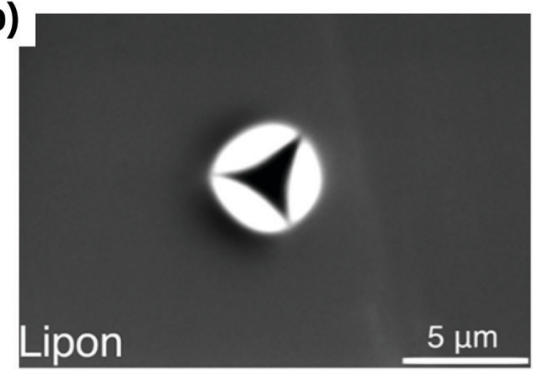

(c)

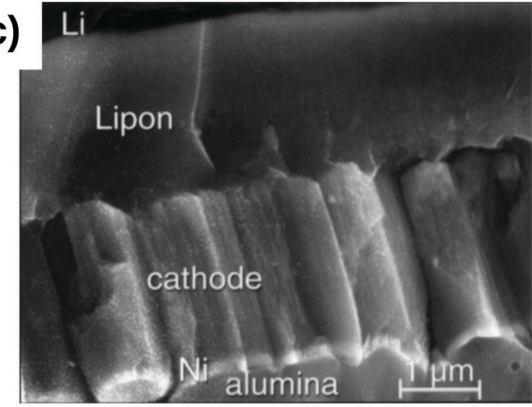

Fig. 7 (a) Stiffness versus fracture toughness of ceramic-based and glassy solid electrolyte. The typical fracture toughness of brittle materials is approximately $1 \mathrm{MPa} \mathrm{m}^{-2}$. When LiPON was subjected to the nanoindentation in the works of Kalnaus et al., ${ }^{61}$ no fracture was observed throughout the experiments. Reprinted from ref. 61, Copyright (2021), with permission from Springer. (b) Under nanoindentation experiments, a significant pile-up instead of cracking was observed in LiPON. Reprinted from ref. 61, Copyright (2021), with permission from Springer. (c) The micrograph of a crosssectional crack observed in LiPON. Reprinted from ref. 62, Copyright (2000), with permission from Elsevier.

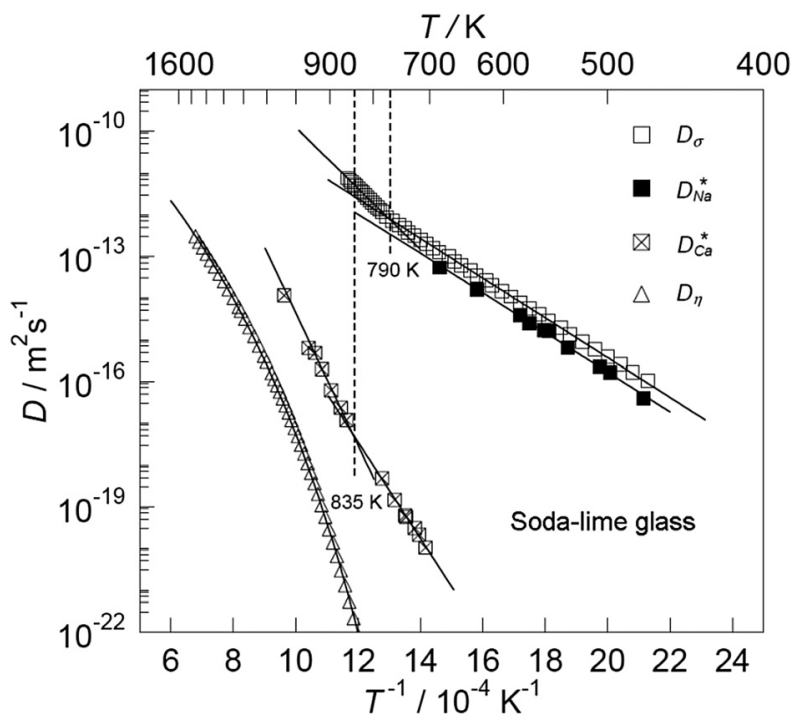

Fig. 8 The temperature-dependent charge diffusion coefficient $\left(D_{\sigma}\right)$, tracer diffusion coefficients of ${ }^{22} \mathrm{Na}\left(D_{\mathrm{Na}}^{*}\right)$ and ${ }^{45} \mathrm{Ca}\left(D_{\mathrm{Ca}}^{*}\right)$ and viscosity diffusion coefficient $\left(D_{\eta}\right)$ for a soda-lime silicate glass. Reproduced from ref. 63, Copyright (2008), with permission from IOP Publishing.

Fig. 9 illustrates the sinusoidal applied strain and the resultant stress response observed in an elastic and a viscoelastic material. ${ }^{56}$ If the material depicts a linearly elastic mechanical behaviour, the strain will be proportional to the stress, as shown by Fig. 9(a). ${ }^{56}$ However, if the material behaves in a viscoelastic manner, a phase shift between the applied strain and resultant stress will be observed, as illustrated by Fig. 9(b). ${ }^{56}$ The loss tangent, $\tan \delta$, is then the ratio between the loss modulus $E^{\prime \prime}[\mathrm{Pa}]$ and the storage modulus $E^{\prime}[\mathrm{Pa}]:^{70,71}$

$$
\tan \delta=\frac{E^{\prime \prime}}{E^{\prime}}
$$

where

$$
\begin{aligned}
& E^{\prime}=\frac{\sigma_{\mathrm{st}, 0}}{\varepsilon_{0}} \cos \delta, \\
& E^{\prime \prime}=\frac{\sigma_{\mathrm{st}, 0}}{\varepsilon_{0}} \sin \delta .
\end{aligned}
$$

Here, $\sigma_{\mathrm{st}, 0}$ and $\varepsilon_{0}$ are the peak amplitudes of stress $\sigma_{\mathrm{st}}$ and strain $\varepsilon$, whereas $\delta$ is the phase shift between stress and strain. ${ }^{70}$ An elastic solid has a loss tangent of zero, indicating that the applied stress and the resulting strain are in phase. In contrast, a viscoelastic solid such as polymers and rubbers can have a loss tangent exceeding unity. ${ }^{53,54,69,72,73}$ Although the loss tangents of inorganic solid electrolytes have not been measured experimentally, similar behaviour can be expected from them as measured in other ceramic materials such as alumina, which has a loss tangent of $9 \times 10^{-4}$ at room temperature. ${ }^{69}$ Compared to the loss tangent of polymers and 
(a)

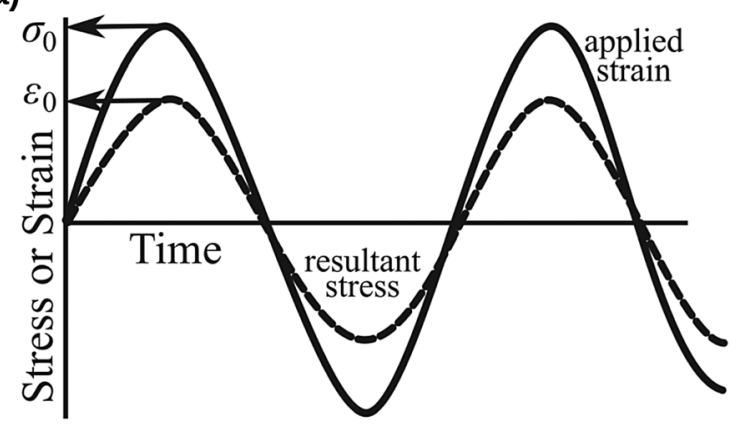

(b)

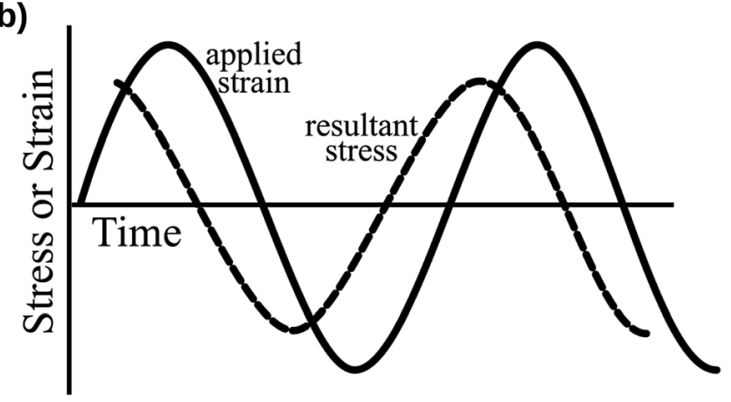

Fig. 9 Sinusoidal applied strain and the resultant stress response observed in (a) an elastic material and (b) a viscoelastic material. Reproduced with permission from ref. 56, Copyright (2012), Princeton University Press.

rubbers, this low magnitude of loss tangent shows that ceramic-based materials exhibit a negligible viscoelastic behaviour at room temperature.

However, as discussed in Section 3.3, due to a large variation in the chemical compositions of inorganic solid electrolytes,${ }^{13}$ we note that the brittle behaviour cannot be generalised for different types of these solid electrolytes. Different classes of inorganic solid electrolytes may exhibit different mechanical behaviour, which requires an individual mechanical characterisation.

\section{Alternatives to Nernst-Einstein's relation}

By using time-resolved operando neutron depth profiling methods, Han et $a l^{74}$ measured the Li concentration profiles in three different representative classes of inorganic solid electrolytes (e.g. LiPON, c-LLZO and $\mathrm{Li}_{3} \mathrm{PS}_{4}$ ) at different temperatures. Their experimental measurements showed a near-zero concentration gradient across the bulk solid electrolytes (see Fig. 10). A near-zero concentration gradient implies that the macroscopic diffusion overpotential in an inorganic solid electrolyte is negligible. ${ }^{60}$ The migration of mobile cations by hopping between lattice sites is the principal conduction mechanism governing the ionic mobility in an inorganic solid electrolyte. ${ }^{60}$ Therefore, the ionic conductivity can be measured directly from the impedance measurement, thus implicitly including the effects of correlations between the mobile cations. ${ }^{6,11,12}$

As material discovery is increasingly aided by computer simulations, there may be materials of interest in which the
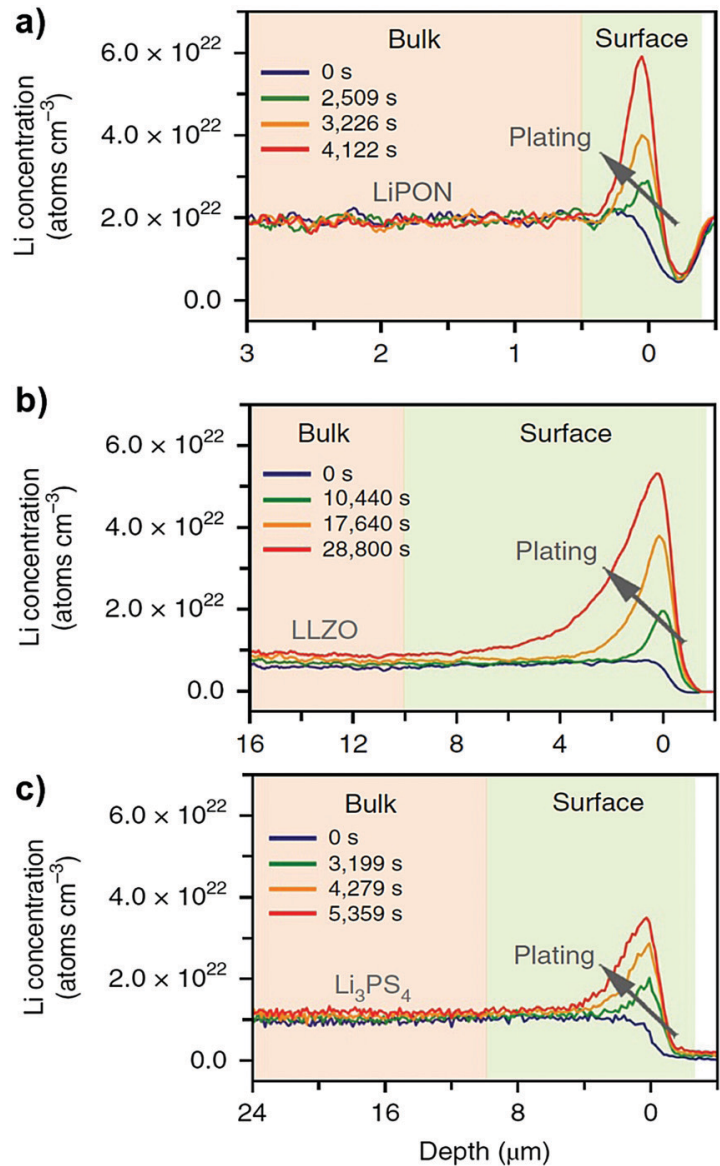

Fig. 10 Measurement of Li concentration profiles using operando neutron depth profiling technique across (a) LiPON (b) c-LLZO and (c) $\mathrm{Li}_{3} \mathrm{PS}_{4}$ solid electrolytes. Reproduced from ref. 74, Copyright (2019), with permission from Springer Nature.

ionic diffusion coefficient is relevant. In such cases, modelling work should focus on the nature of governing forces affecting the ionic mobility through quantum mechanical calculations and force-fields simulations.

For a ceramic-based solid electrolyte, the properties of a ceramic and how its atoms are arranged in its structure are determined predominantly by the nature and the directionality of the bonds holding the atoms together. ${ }^{75}$ In general, two types of bonds are governing the behaviours of ceramics: ionic and covalent bonds. ${ }^{75,76}$ Ionic bonding is an electrostatic force of attraction that results from the electrons transfer between a metal and a non-metal. ${ }^{75,76}$ Ionic compounds are typically brittle and have poor electrical and thermal conductivity. ${ }^{75,76}$ On the other hand, covalent bonding occurs due to sharing of outer shell electrons between two non-metals that have similar electronegativity. ${ }^{75,76}$ Although both types of bonds are present in ceramics, ionic bonding is more dominant, and the fundamental force governing the atomic structure in ceramics is the electrostatic Coulombic force ${ }^{75,76}$ By using appropriate boundary conditions, Schrödinger's equation can be solved considering the dynamic equilibrium between various governing forces such as electrostatic Coulombic force and the forward electric 
force. ${ }^{75}$ The outcome of such investigations could determine whether Nernst-Einstein's relation can be applied for inorganic solid electrolytes or a new relation is required to describe the interatomic forces acting in these electrolytes.

Depending on the structure and the chemical composition of the host lattice, the atomic diffusion in a solid can be described by a range of diffusion mechanisms, which include interstitial mechanisms, collective mechanisms, vacancy mechanisms, divacancy mechanisms or interstitial-substitutional exchange mechanisms. ${ }^{64}$ For glassy solid electrolytes, atomistic simulation can be implemented to evaluate which diffusion mechanisms best describe the hopping behaviour observed experimentally in the solid electrolyte. By studying the relationship between mean displacement and time for colloidal particles, Jean Perrin and his students had performed a series of experiments in 1908 to evaluate Einstein's and EinsteinSmoluchowski's relation. ${ }^{33}$ Instead of assuming the general applicability of Nernst-Einstein's relation for all electrolytes, the Jean Perrin's experiments can be extended to evaluate whether the Einstein's diffusion model can describe the hopping mechanisms for different types of inorganic solid electrolytes.

\section{Conclusions}

In solid-state battery research, Nernst-Einstein's model has often been used to relate the diffusion coefficient of mobile cations to the electrolyte conductivity. However, a near-zero concentration gradient across the inorganic solid electrolyte indicates that the diffusion overpotential is insignificant, and the migration of mobile cations is the primary conduction mechanism. In this case, ionic conductivity can be measured from the impedance measurement of inorganic solid electrolytes, where the ion-ion correlations are also considered implicitly through a direct experimental measurement.

We show that the popular Nernst-Einstein's relation is not valid for all materials. Instead of using by default NernstEinstein's relation originally developed for fluids, future studies should calculate the ionic mobility from quantum mechanical or force-field simulations. Such approach could determine how the nature of governing forces affect the ionic mobility in an inorganic solid electrolyte. Then, the calculated ionic mobility can be used in cell- and system-level solid-state battery modelling to predict battery performance.

We also highlight that the measurement of loss tangent is necessary to characterise the viscoelastic behaviour of inorganic solid electrolytes. If the loss tangent of inorganic solid electrolytes is less than unity in the range of batteries operating temperatures, the impacts of using Nernst-Einstein's relation in modelling the ionic mobility in inorganic solid electrolytes must be evaluated before further use. A new relation describing the governing forces is required. However, if the measured loss tangent is comparable to that of polymers and $\mathrm{Li}$ metal, the viscoelastic behaviour of inorganic solid electrolytes should be quantified.

\section{Conflicts of interest}

There are no conflicts of interest to declare.

\section{Acknowledgements}

Much appreciated is the strong support received from Beijing Institute of Aeronautical Materials (BIAM). The research was performed at the BIAM-Imperial Centre for Materials Characterisation, Processing and Modelling at Imperial College London.

\section{References}

1 J. Janek and W. G. Zeier, Nat. Energy, 2016, 1, 1167.

2 Y. Kato, S. Hori, T. Saito, K. Suzuki, M. Hirayama, A. Mitsui, M. Yonemura, H. Iba and R. Kanno, Nat. Energy, 2016, 1, 652.

3 Y.-H. Chiang, W.-Y. Sean and J.-C. Ke, J. Power Sources, 2011, 196, 3921-3932.

4 S. Alavi, C. R. Birkl and D. A. Howey, J. Power Sources, 2015, 288, 345-352.

5 J. Li, C. Ma, M. Chi, C. Liang and N. J. Dudney, Adv. Energy Mater., 2015, 5, 1401408.

6 S. D. Fabre, D. Guy-Bouyssou, P. Bouillon, F. Le Cras and C. Delacourt, J. Electrochem. Soc., 2012, 159, A104.

7 C. Loho, R. Djenadic, M. Bruns, O. Clemens and H. Hahn, J. Electrochem. Soc., 2017, 164, A6131-A6139.

8 D. Rettenwander, G. Redhammer, F. Preishuber-Pflügl, L. Cheng, L. Miara, R. Wagner, A. Welzl, E. Suard, M. M. Doeff, M. Wilkening, J. Fleig and G. Amthauer, Chem. Mater., 2016, 28, 2384-2392.

9 J. B. Bates, N. J. Dudney, G. R. Gruzalski, R. A. Zuhr, A. Choudhury, C. F. Luck and J. D. Robertson, J. Power Sources, 1993, 43, 103-110.

10 P. Braun, C. Uhlmann, M. Weiss, A. Weber and E. IversTiffée, J. Power Sources, 2018, 393, 119-127.

11 L. Cheng, W. Chen, M. Kunz, K. Persson, N. Tamura, G. Chen and M. Doeff, ACS Appl. Mater. Interfaces, 2015, 7, 2073-2081.

12 S. Larfaillou, D. Guy-Bouyssou, F. Le Cras and S. Franger, J. Power Sources, 2016, 319, 139-146.

13 J. C. Bachman, S. Muy, A. Grimaud, H.-H. Chang, N. Pour, S. F. Lux, O. Paschos, F. Maglia, S. Lupart, P. Lamp, L. Giordano and Y. Shao-Horn, Chem. Rev., 2016, 116, 140-162.

14 A. Bates, S. Mukherjee, N. Schuppert, B. Son, J. G. Kim and S. Park, Int. J. Energy Res., 2015, 39, 1505-1518.

15 D. Danilov, R. A. H. Niessen and P. H. L. Notten, J. Electrochem. Soc., 2011, 158, A215.

16 N. Kazemi, D. L. Danilov, L. Haverkate, N. J. Dudney, S. Unnikrishnan and P. H. Notten, Solid State Ionics, 2019, 334, 111-116.

17 N. J. J. de Klerk, E. van der Maas and M. Wagemaker, ACS Appl. Energy Mater., 2018, 1, 3230-3242.

18 A. M. Nolan, Y. Zhu, X. He, Q. Bai and Y. Mo, Joule, 2018, 2, 2016-2046.

19 S. Ohno, A. Banik, G. F. Dewald, M. A. Kraft, T. Krauskopf, N. Minafra, P. Till, M. Weiss and W. G. Zeier, Progr. Energy, 2020, 2, 022001.

20 X. Song, Y. Lu, F. Wang, X. Zhao and H. Chen, J. Power Sources, 2020, 452, 227803. 
21 A. A. Talin, D. Ruzmetov, A. Kolmakov, K. McKelvey, N. Ware, F. El Gabaly, B. Dunn and H. S. White, ACS Appl. Mater. Interfaces, 2016, 8, 32385-32391.

22 H.-K. Tian and Y. Qi, J. Electrochem. Soc., 2017, 164, E3512-E3521.

23 H.-K. Tian, A. Chakraborty, A. A. Talin, P. Eisenlohr and Y. Qi, J. Electrochem. Soc., 2020, 167, 090541.

24 M.-C. Pang, Mechanical behaviour of inorganic solid-state batteries: Can we model the ionic mobility in the electrolyte with NernstEinstein's relation? 2021, DOI: 10.5281/zenodo.5559668.

25 A. Marcolongo and N. Marzari, Phys. Rev. Mater., 2017, 1(2), 025402.

26 N. M. Vargas-Barbosa and B. Roling, ChemElectroChem, 2020, 7, 367-385.

27 A. Einstein, Investigations on the theory of the Brownian movement: on the movement of small particles suspended in a stationary liquid demanded by the molecular kinetic theory of heat, Dover Publications, 1956, pp. 1-18.

28 C. M. A. Brett and A. M. O. Brett, Electrochemistry: Principles, methods, and applications/Christopher M.A. Brett and Ana Maria Oliveira Brett, Oxford University Press, Oxford, 1993.

29 R. P. Feynman, R. B. Leighton and M. L. Sands, The Feynman lectures on physics, New millennium edn, Basic Books, New York, 2011, p. 2010.

30 G. Peskir, Stochastic Models, 2003, 19, 383-405.

31 J. S. Newman and K. E. Thomas-Alyea, Electrochemical systems, 3rd edn, Wiley, Hoboken, NJ and Chichester, 2004.

32 M. Islam, Phys. Scr., 2004, 70, 120.

33 M. D. Haw, J. Phys.: Condens. Matter, 2002, 14, 7769.

34 G. E. Murch, Diffusion Kinetics in Solids, John Wiley \& Sons, Ltd, 2001, ch. 3, pp. 171-238.

35 K. Hayamizu, Y. Aihara, S. Arai and C. G. Martinez, J. Phys. Chem. B, 1999, 103, 519-524.

36 Y. Aihara, K. Sugimoto, W. S. Price and K. Hayamizu, J. Chem. Phys., 2000, 113, 1981-1991.

37 K. Hayamizu and Y. Aihara, Solid State Ionics, 2013, 238, 7-14.

38 K. Hayamizu, Y. Aihara and W. S. Price, J. Chem. Phys., 2000, 113, 4785-4793.

39 K. Hayamizu, Y. Terada, K. Kataoka, J. Akimoto and T. Haishi, Phys. Chem. Chem. Phys., 2019, 21, 23589-23597.

40 K. Hayamizu, Y. Terada, K. Kataoka and J. Akimoto, J. Chem. Phys., 2019, 150, 194502.

41 K. Hayamizu, Y. Aihara, S. Arai and W. S. Price, Solid State Ionics, 1998, 107, 1-12.

42 H. Tokuda, K. Ishii, M. A. B. H. Susan, S. Tsuzuki, K. Hayamizu and M. Watanabe, J. Phys. Chem. B, 2006, 110, 2833-2839.

43 A. Noda, K. Hayamizu and M. Watanabe, J. Phys. Chem. B, 2001, 105, 4603-4610.

44 S. Yu, R. D. Schmidt, R. Garcia-Mendez, E. Herbert, N. J. Dudney, J. B. Wolfenstine, J. Sakamoto and D. J. Siegel, Chem. Mater., 2016, 28, 197-206.

45 A. Sakuda, A. Hayashi, Y. Takigawa, K. Higashi and M. Tatsumisago, J. Ceram. Soc. Jpn., 2013, 121, 946-949.

46 J. E. Ni, E. D. Case, J. S. Sakamoto, E. Rangasamy and J. B. Wolfenstine, J. Mater. Sci., 2012, 47, 7978-7985.
47 J. Wolfenstine, H. Jo, Y.-H. Cho, I. N. David, P. Askeland, E. D. Case, H. Kim, H. Choe and J. Sakamoto, Mater. Lett., 2013, 96, 117-120.

48 F. P. McGrogan, T. Swamy, S. R. Bishop, E. Eggleton, L. Porz, X. Chen, Y.-M. Chiang and K. J. van Vliet, Adv. Energy Mater., 2017, 7, 1602011.

49 J. A. Lewis, F. J. Q. Cortes, M. G. Boebinger, J. Tippens, T. S. Marchese, N. Kondekar, X. Liu, M. Chi and M. T. McDowell, ACS Energy Lett., 2019, 4, 591-599.

50 G. A. Gogotsi, in Encyclopedia of Thermal Stresses, ed. R. B. Hetnarski, Springer Netherlands, Dordrecht, 2014, pp. 497-505.

51 S. Korte and W. J. Clegg, Scr. Mater., 2009, 60, 807-810.

52 J. D. Ferry, Viscoelastic properties of polymers, 3rd edn, Wiley, New York and Chichester, 1980.

53 H. Watanabe, Prog. Polym. Sci., 1999, 24, 1253-1403.

54 H. K. Nguyen, M. Ito, S. Fujinami and K. Nakajima, Macromolecules, 2014, 47, 7971-7977.

55 A. Masias, N. Felten, R. Garcia-Mendez, J. Wolfenstine and J. Sakamoto, J. Mater. Sci., 2019, 54, 2585-2600.

56 J. Vincent, Structural biomaterials, Princeton University Press, 2012, pp. 1-28.

57 Z. Wang, J. Z. Lee, H. L. Xin, L. Han, N. Grillon, D. GuyBouyssou, E. Bouyssou, M. Proust and Y. S. Meng, J. Power Sources, 2016, 324, 342-348.

58 R. Chen, A. M. Nolan, J. Lu, J. Wang, X. Yu, Y. Mo, L. Chen, X. Huang and H. Li, Joule, 2020, 4, 812-821.

59 Z. Wang, D. Santhanagopalan, W. Zhang, F. Wang, H. L. Xin, K. He, J. Li, N. Dudney and Y. S. Meng, Nano Lett., 2016, 16, 3760-3767.

60 M.-C. Pang, K. Yang, R. Brugge, T. Zhang, X. Liu, F. Pan, S. Yang, A. Aguadero, B. Wu and M. Marinescu, et al., Mater. Today, 2021, DOI: 10.1016/j.mattod.2021.02.011.

61 S. Kalnaus, A. S. Westover, M. Kornbluth, E. Herbert and N. J. Dudney, J. Mater. Res., 2021, 1-10.

62 J. B. Bates, N. J. Dudney, B. Neudecker, A. Ueda and C. D. Evans, Solid State Ionics, 2000, 135, 33-45.

63 H. Mehrer, A. W. Imre and E. Tanguep-Nijokep, J. Phys.: Conf. Ser., 2008, 106, 012001.

$64 \mathrm{H}$. Mehrer, Diffusion in solids: fundamentals, methods, materials, diffusion-controlled processes, Springer Science \& Business Media, 2007, vol. 155.

65 G. Fan, H. Choo and P. Liaw, J. Non-Cryst. Solids, 2007, 353, 102-107. 66 E. T. Njiokep and H. Mehrer, Solid State Ionics, 2006, 177, 2839-2844.

67 M. D. Demetriou, M. E. Launey, G. Garrett, J. P. Schramm, D. C. Hofmann, W. L. Johnson and R. O. Ritchie, Nat. Mater., 2011, 10, 123-128.

68 X. Xi, D. Zhao, M. Pan, W. Wang, Y. Wu and J. Lewandowski, Phys. Rev. Lett., 2005, 94, 125510.

69 R. S. Lakes, Viscoelastic solids: Viscoelastic properties of materials, CRC Press, Boca Raton, Fla. and London, 1999.

70 F. K. Fuss, in Nonlinear approaches in engineering applications, ed. L. Dai and R. N. Jazar, Springer, Cham, 2014, pp. 137-157.

71 J.-J. Chang, Y.-Y. Li, X.-F. Zeng, H.-Y. Zhong, T.-L. Wan and C. Lu, Materials, 2019, 12, 1-15. 
72 C. G. Robertson and M. Rackaitis, Macromolecules, 2011, 44, 1177-1181.

73 J. D. Ferry, Macromolecules, 1991, 24, 5237-5245.

74 F. Han, A. S. Westover, J. Yue, X. Fan, F. Wang, M. Chi, D. N. Leonard, N. J. Dudney, H. Wang and C. Wang, Nat. Energy, 2019, 4, 187-196.
75 M. Barsoum, Fundamentals of ceramics, CRC press, 2019.

76 The American Ceramic Society, Structure and Properties of Ceramics, available at https://ceramics.org/about/what-areengineered-ceramics-and-glass/structure-and-properties-ofceramics (2021/04/02). 